\title{
Lower-limb joint work and power are modulated during load carriage based on load configuration and walking speed
}

Submitted as an Original Article

\section{Corresponding author}

${ }^{\mathrm{a}}$ Gavin K Lenton

g.lenton@griffith.edu.au

Phone: +61433334176

${ }^{\mathrm{b}}$ Tim L.A Doyle

tim.doyle@mq.edu.au

${ }^{\mathrm{a}}$ David G Lloyd

david.lloyd@griffith.edu.au

ajeremy Higgs

Jeremy.higgs@griffithuni.edu.au

${ }^{\mathrm{c}}$ Daniel Billing

daniel.billing@dst.defence.gov.au

${ }^{\mathrm{a}}$ David J Saxby

d.saxby@griffith.edu.au

${ }^{a}$ Gold Coast Orthopaedics Research, Engineering and Education Alliance, Menzies Health Institute Queensland, School of Allied Health Sciences, Griffith University, 58 Parklands Drive, Southport, Queensland, 4215, Australia

${ }^{\mathrm{b}}$ Department of Health Professions, Faculty of Medicine and Health Sciences, Macquarie University, Balaclava Road, North Ryde, New South Wales 2109

${ }^{c}$ Land Division, Defence Science and Technology Group, 506 Lorimer Street, Fishermans Bend, VIC 3207

\section{Keywords}

Load carriage

Joint power

Musculoskeletal modelling

Military

Walking

Word count: 3908 


\section{Abstract}

Soldiers regularly transport loads weighing $>\mathbf{2 0} \mathrm{kg}$ for slow speeds over long durations. These tasks elicit high energetic costs through increasing positive work generated by knee and ankle muscles, which may increase risk of muscular fatigue and decrease combat readiness. This study aimed to determine how modifying where load is borne changes lower-limb joint mechanical work production, and if load magnitude and/or walking speed also affect work production. Twenty Australian soldier participants donned a total of 12 body armor variations: six body armor systems (one standard-issue, two commercially available [cARM1-2], and three prototype [pARM1-3]) and two load magnitudes (15 and 30 $\mathrm{kg})$. In each armor variation, participants completed treadmill walking at two speeds (1.51 and $1.83 \mathrm{~m} / \mathrm{s})$. Three-dimensional motion capture and force plate data were acquired and used to estimate joint angles and moments from inverse kinematics and dynamics, respectively. From these, hip, knee, and ankle joint work was computed and compared between armor types and walking speeds. Positive joint work over the stance phase significantly increased with walking speed and carried load, accompanied by 2.3$2.6 \%$ shifts in total positive work production from ankle to hip $(\mathrm{p}<0.05)$. Compared to using cARM1 with $15 \mathrm{~kg}$ of carried load, carrying $30 \mathrm{~kg}$ caused significantly greater hip contribution to total positive work, while knee and ankle work decreased. Substantial increases in hip joint contributions to total positive work with increases in walking speed and load magnitude highlight the importance of hip musculature to positive power generation in load carriage walking. 


\section{Introduction}

Depending on training and operational requirements, soldiers transport equipment purposefully at high speed and slowly over long durations (e.g., 4-5 hours). In particular, long duration military load carriage tasks impose substantial metabolic costs that affect soldiers' in-field capabilities (Beekley et al., 2007; Huang and Kuo, 2014). It has been shown that net metabolic energy rate increases proportionally with carried load during simulated marching (Quesada et al., 200o), which can decrease time to fatigue, the capacity of soldiers to maintain a given work intensity (Johnson et al., 1995), and soldier combat readiness (e.g., ability to perform explosive movements) following marching (Knapik et al., 1997). Therefore, identifying the most efficient means to carry load would benefit soldiers operating in the field.

Walking and carrying load increases metabolic cost potentially through increased muscle contractionrelated consumption of energy substrates (Umberger and Rubenson, 2011). Indeed, studies have shown positive mechanical work increases approximately linearly with metabolic expenditure during loaded walking (Huang and Kuo, 2014; Pierrynowsi et al., 1981). Mechanical work performed by the human body describes the change in, or amount of, mechanical energy transmitted by muscle or external force(s) over time (Robertson et al., 2013). Muscles producing force during shortening (i.e., concentric) generate positive work, while during lengthening contractions (i.e., eccentric) muscles do negative work (Donelan et al., 2002). Recent studies have reported total and joint-specific work and powers increase in response to increased carried load during walking (Grenier et al., 2012; Huang and Kuo, 2014; Wang et al., 2013, Panizzolo et al. 2016) and running (Liew et al., 2016) tasks. In these studies, hip muscles perform positive work to stabilize the large moment of inertia of the combined upper body and equipment mass and swing the trailing leg (Winter and Eng, 1995). Knee muscles perform greater positive work in rebound following ground impact and contribute to raising the body COM (Huang and Kuo, 2014). Ankle muscles provide positive work during late stance to propel the body centre of mass (COM) forward, which also is a feature of unloaded gait as walking speed increases (Umberger and Martin, 2007). A limitation of previous studies is that only moderate loads ( $15-20 k g)$ isolated to one portion of a backpack or COM were carried at relatively slow walking speeds, or exclusively during

running. Additionally, the one study to use military-specific loads did not derive joint work using inverse dynamics, which is believed to better represent musculotendon work compared to calculating external COM work (Zelik and Kuo, 2012). Research is therefore required to examine how carried load and walking speed influence positive mechanical work and power during simulated marching in soldiers.

Simpler solutions to assist load carriage, such as incorporating a hip belt into body armor, could redistribute positive power to reduce metabolic cost. A load sharing system is defined as a body armor system integrating a torso vest and hip belt (connected by struts) that changes the part of the body on which the mass is carried: in our case, the load sharing system transfers the load from primarily the 
shoulders to both the shoulders and hips. Redistributing load toward the hips could increase contributions of hip muscles to forward progression of the body COM and preserve normal knee mechanical power because relative moments of inertia would be lower with load closer to hips (Winter et al., 1990). In unloaded walking, $>80 \%$ of average positive joint power per stride has been shown to come from the ankle ( $\sim 5 \%$ ) and hip ( $45 \%)$ joints (Farris and Sawicki, 2012; Rubenson et al., 2011; Umberger and Martin, 2007). This indicates positive work is generated primarily by ankle plantarflexors and hip flexors/extensors. When walking speed is increased, studies have shown the magnitude of each joint and/or muscle's contribution to positive power increases proportionally, with relative contributions remaining similar (Dorn et al., 2015; Farris and Sawicki, 2012; Schache et al., 2015). Theoretically, an individual would similarly 'scale-up' joint positive powers as a neuromuscular strategy to compensate for increasing carried load and/or walking speed, yet this remains to be investigated. During physically demanding tasks (e.g., load carriage walking), joint power could shift from an ankle dominant strategy to a hip dominant strategy, because hip muscles can generate larger moments and powers compared with ankle muscles. However, shifting power production to proximal joints would increase active work performed by muscle as hip-spanning gluteal muscles have very short tendons (Neptune et al., 2009) compared to Achilles tendon which has advantageous elastic properties (Lichtwark and Wilson, 2008). Importantly, less reliance on knee muscles to generate positive power could reduce time to fatigue during load carriage compared to relying on ankle and hip muscles, which have been shown to be less susceptible to fatigue during load carriage (Blacker et al., 2013). Thus, a proximal shift in joint powers would have important implications for soldier performance, device design, and physical training prescription, but has yet to be investigated.

This study examined how lower-limb joint work and power magnitudes are modulated in different load configurations and walking speeds during gait. Load configuration includes both the body armor type and load magnitude. A secondary aim was to determine whether relative contributions of lower-limb joints to total mechanical positive power were affected by different load configurations and walking speeds. We hypothesized using load sharing systems would increase total positive work and positive hip work compared to not using load sharing systems. Additionally, we hypothesized increasing carried load or walking speed would increase positive joint work and power magnitudes. Finally, we hypothesized relative joint contributions to total mechanical positive power was similar for different carried loads and/or walking speeds, but a shift towards the hips would occur when using load sharing systems compared to no load sharing.

\section{Methods}

\section{Participants}

Twenty-one male soldiers (age: $29.5 \pm 7.1$ years, height: $1.77 \pm 0.08 \mathrm{~m}$, mass $82.8 \pm 12.1 \mathrm{~kg}$ ) participated and at the time of testing no participants had acute or chronic injuries. Participants gave their written 
informed consent to the protocol, which was approved by Australian Defence Human Research Ethics Committee (Protocol 756-14).

\section{Body Armor Conditions}

Five different load sharing systems: two commercial (cARM1, cARM2) and three prototype (pARM1, pARM2, pARM3) designs were tested against the current Australian Army standard-issue Tiered Body Armor System (TBAS) in two operational configurations (15 kg and $30 \mathrm{~kg}$ ). The $15 \mathrm{~kg}$ load included two replica ballistic plates and soft armor inserts, as well as replica equipment (e.g., radio, ammunition) placed in pouches around the vest. For the $30 \mathrm{~kg}$ configuration, an assault pack was attached at vest rear and contained $15 \mathrm{~kg}$ of load evenly distributed throughout the pack. Descriptions and illustrations of the armor types can be found in the supplementary material. The effectiveness of each load sharing system in reducing shoulder pressure has been established in our previous work (Lenton et al., 2018).

\section{Procedures}

Participants completed a standardized treadmill walking protocol for each of the 12 armor $\times$ load conditions (i.e., six body armor systems and two loads), as well as a baseline no-armor condition across four laboratory-based testing sessions. The baseline condition, in which participants wore a compression shirt, standard athletic shorts, and their own military boots, was always completed first, while the order of wearing other armor conditions was randomised and counterbalanced. The walking protocol consisted of a 5 -minute warm-up at $1.25 \mathrm{~m} \cdot \mathrm{s}^{-1}, 10$-minutes walking at $1.53 \mathrm{~m} \cdot \mathrm{s}^{-1}$ (i.e., moderate), 10-minutes walking at $1.81 \mathrm{~m} \cdot \mathrm{s}^{-1}$ (i.e., fast), and a 5-minute cool down at $1.25 \mathrm{~m} \cdot \mathrm{s}^{-1}$. Following completion of walking tasks, participants rested for a minimum of 25-minutes and completed subsequent armor conditions for the session.

To allow measurement of 3-dimensional motion, spherical, $14 \mathrm{~mm}$ diameter retro-reflective markers, and marker clusters were placed on the torso, and bilaterally on the head, arms, and legs of participants according to a marker set we previously developed and validated (Lenton et al., 2017). Marker and GRF data were concurrently and synchronously acquired using an 11-camera, 3-dimensional motion capture system (Vicon, Oxford, UK) and a split-belt, force-sensing treadmill (AMTI Compact Tandem, Watertown, MA, USA). Prior to treadmill walking, static standing calibration and pointer trials were acquired to measure 3-dimensional positions of 14 markers, which were later used to define joint centres and track segments during dynamic tasks. Marker positions, sampled at $100 \mathrm{~Hz}$, and ground reaction forces (GRFs), sampled at $1000 \mathrm{~Hz}$, were acquired during treadmill walking for a 30-second epoch in the final minute of each walking speed.

\section{Data Processing}

Raw marker trajectories from static and dynamic trials were cleaned using Vicon Nexus version 2.3. Cleaned trials were then exported to Matlab and processed using a custom implementation of biomechanical processing software (R2014b, The Mathworks) (Mantoan et al., 2015). Processing 
involved partitioning 30 second data epochs into individual right limb gait cycles, digital filtering of marker trajectories and GRFs using a using a $4^{\text {th }}$ order zero-lag (Robertson and Dowling, 2003) Butterworth filter at $10 \mathrm{~Hz}$, and transforming data from the laboratory coordinate system to model coordinate system used in OpenSim (Delp et al., 2007).

\section{Biomechanical modelling}

For each participant, a generic scaled, full-body anatomic model (Rajagopal et al., 2016) was used to represent the head, torso, arms, pelvis, and lower-limb. The model included a six DOF pelvis, a three rotational DOF hip joint, and one rotational DOF knee and ankle joints. For the knee, non-sagittal rotations and tibial translations were parameterized as functions of knee flexion (Walker et al., 1988). Scaled anatomic models were then used in OpenSim inverse kinematics (IK) analysis to determine model kinematics (Reinbolt et al., 2005), and inverse dynamics analysis to compute net moments at the right hip, knee, and ankle joints. Ensemble-averaged data for a minimum of 10 gait cycles (ranging from 10-28 gait cycles) from each $30 \mathrm{~s}$ data epoch were created for each participant.

Sagittal-plane hip, knee, and ankle joint power was determined by the dot product of joint moments and angular velocities (Rubenson et al., 2011), and normalized to each participant's body mass $\left(\mathrm{Wkg}^{-1}\right)$. Instantaneous joint power curves were split into power phases previously defined across the gait cycle (Winter, 1987), whereby positive phases represented periods of energy generation and negative phases represented energy absorption. Numerical integration of instantaneous joint power curves within the defined phases enabled calculation of positive $\left(W_{j}^{+}\right)$and negative $\left(W_{j}^{-}\right)$joint work $\left(\mathrm{Jkg}^{-1}\right)$, which was multiplied by two to approximate output from both limbs. Total right limb positive $W_{\text {tot }}^{+}$and negative $W_{\text {tot }}^{-}$work were determined by summing positive and negative hip, knee, and ankle joint work, respectively. Positive and negative work terms were divided by stride cycle time to give positive $\left(\dot{W}_{\text {tot }}^{+}\right)$ and negative $\left(\dot{W}_{\text {tot }}^{-}\right)$mass-specific mechanical power terms $\left(\mathrm{Wkg}^{-1}\right)$ (Rubenson et al., 2011). These terms represented energy generated and absorbed by the limbs during an average stride. This analysis was performed separately for stance and swing phases of the gait cycle, which were defined based on heelstrike and toe-off gait events. To compare relative contributions of each joint towards $W_{\text {tot }}^{+}$and $W_{\text {tot }}^{-}$, each joint's $W_{j}^{+}$and $W_{j}^{-}$was expressed as a percentage of $W_{\text {tot }}^{+}$and $W_{\text {tot }}^{-}$, respectively. Additionally, power data during load carriage were normalized relative to the baseline unloaded condition for each walking speed.

Absolute and relative total lower-limb and joint-specific positive and negative mechanical power per stride were averaged between participants for every armor type, load, and walking speed for statistical analyses. Although data from pARM2 and $\mathrm{pARM}_{3}$ armor systems were collected, it was not included in statistical analyses because these designs had very similar design features to PARM1, but were very new prototypes while pARM1 was a mature prototype, and statistical comparisons of outcomes variables between the pARM1-3 armor systems revealed no significant differences. 


\section{Statistical Analysis}

Statistical analysis was performed using $\mathrm{R}$ version 3.3.1 in R-Studio (RStudio, Inc, Boston, MA) vo.99.903. Outliers were removed using Tukey's method (Hoaglin et al., 1986) and missing data were imputed using predictive mean matching (Buuren and Groothuis-Oudshoorn, 2011) to generate a complete dataset for statistical comparisons ( $<5 \%$ data missing for any variable) (Royston, 2004). Shapiro-Wilk tests confirmed normally distributed data, and three-way repeated measures ANOVAs tested for significant interactions between, and main effects of, armor type, carried load, and walking speed. Post-hoc paired t-tests were performed on significant interactions or main effects to identify specific differences with p-values adjusted to control for multiple comparisons (Benjamini and Hochberg, 1995). Significance was set at $p<0.05$. Effect sizes (i.e., partial $\eta^{2}$ ) were calculated for all the significant main and interaction effects, with small, medium, and large effects defined as $\eta^{2}$ between 0.01 and $0.06,0.06$ and 0.14 , and greater than 0.14, respectively (Richardson, 2011). Linear regression was used to test how hip, knee, and ankle positive mechanical power in stance changed as a function of total mechanical positive power. Each regression was performed with a single slope across all participants, but with separate slopes for each load and walking speed combination.

\section{Results}

\section{Effects of wearing different armor types}

When expressed relative to results obtained in the baseline condition, wearing any armor type increased total joint-specific positive power over the stance phase (Table 1, Table 2), but there were no significant differences between armor types ( $>0.05$ ). Significant main effects of armor type were observed for total positive power $\left(\mathrm{p}<0.05, \eta_{p}^{2}=0.06\right)$ and positive hip power over the swing phase $\left(\mathrm{p}<0.05, \eta_{p}^{2}=0.06\right)$. Specifically, compared to TBAS, positive hip power during swing increased $14.3 \%$, $14.3 \%, 18.2 \%$ when wearing cARM1, cARM2, and pARM, respectively. Hip and ankle joints were responsible for generating most of the power during the stance phase of gait (Figure 1). A significant interaction was found between load sharing systems and load magnitude for the relative contribution of the hip to total mechanical positive power $\left(\mathrm{p}=0.013, \eta_{p}^{2}=0.03\right)$. Specifically, the hip contributed more to total mechanical positive power with $30 \mathrm{~kg}$ compared to $15 \mathrm{~kg}$ of carried load for cARMı armor type $(\mathrm{p}<0.05)$.

\section{INSERT TABLE 1 ABOUT HERE}

INSERT FIGURE 1 ABOUT HERE

\section{Effects of increasing load magnitude and/or walking speed}

Significant main effects of load and walking speed were found for total and joint-specific positive power (Table 2), with absolute magnitudes increasing when walking faster or carrying more load (Figure 2). Total positive power performed in stance was sensitive to increases in carried load $\left(\eta_{p}^{2}=0.19\right)$ and walking speed $\left(\eta_{p}^{2}=0.17\right)$. Additionally, there were significant main effects of load for positive power 
generated at the hip, knee, and ankle ( $\mathrm{p}<0.05)$ relative to unloaded walking. Compared to carrying $15 \mathrm{~kg}$ of load, carrying $30 \mathrm{~kg}$ of load resulted in significantly greater positive power at the hip $(87.1 \pm 4.3 \% \mathrm{vs}$. $57.9 \pm 4.3 \%)$, knee (69.3 $\pm 6.0 \%$ vs. $37.8 \pm 5.7 \%)$, and ankle (40.2 $\pm 2.4 \%$ vs. $26.1 \pm 2.2 \%)$.

\section{INSERT TABLE 2 ABOUT HERE}

Hip and knee power increased with increased total positive joint power $(\mathrm{p}<0.05)$, whereas ankle power did not (Figure 3). The correlation coefficient was much greater for hip power $\left(R^{2}=0.66\right)$ than for knee power $\left(\mathrm{R}^{2}=0.33\right)$, indicating the hip was primarily responsible for generating more positive power with increasing task demands. Additionally, compared to walking without load, the hip joint generated $14.9 \pm 1.6 \%$ and $37.6 \pm 1.9 \%$ more power in early swing phase when participants carried $15 \mathrm{~kg}$ and $30 \mathrm{~kg}$, respectively.

INSERT FIGURE 2 ABOUT HERE

A main effect of walking speed ( $\mathrm{p}=\mathrm{o.01}, \eta_{p}^{2}=\mathrm{o.02}$ ) was observed for percent contribution of the hip joint to total mechanical positive power. Additionally, significant main effects of walking speed ( $\mathrm{p}<0.05$, $\eta_{p}^{2}=0.03$ ) and load ( $\mathrm{p}=\mathrm{0.04}, \eta_{p}^{2}=\mathrm{o.01}$ ) were found for the percent contribution of the ankle to total mechanical positive power. When increasing walking speed from moderate to fast, hip contribution to total mechanical positive power increased $2.3 \%$, while ankle contribution decreased $2.6 \%$. A similar decrease in ankle contribution was observed when carried load increased, but was offset by small increases in hip and knee contributions.

\section{INSERT FIGURE 3 ABOUT HERE}

\section{Discussion}

The purpose of this study was to examine the effects of redistributing torso-borne load, carried load magnitude, and walking speed on total and joint lower-limb power in soldiers during military-specific walking. This study also explored whether load redistribution, load magnitude, and walking speed influenced hip, knee, and ankle contributions to total positive power, and considered these strategies indicative of neuromuscular coordination. Full-body motion and GRFs were measured from soldiers as they completed treadmill walking at moderate and fast speeds. All participants completed testing with six different body armor systems (although only data for only four were presented) and two (15 kg and $30 \mathrm{~kg}$ ) load configurations. Using an inverse kinematics and dynamics approach, total and joint lowerlimb positive and negative instantaneous mechanical power per stride were determined and compared between experimental conditions.

Contrary to our first hypothesis, no significant differences in stance phase power generation or absorption were observed between load sharing systems, despite a main effect existing for hip power generation (Table 1). This result, along with our prior work demonstrating all of the load sharing systems were effective in reducing shoulder pressure (Lenton et al., 2018), means that shoulder-to-hip load transfer likely does not require greater muscular effort during walking compared to standard 
beltless designs. Consistent with prior research (Devroey et al., 2007), these findings suggest changes in coordination and energetics mostly occur when load is positioned close to extremities (e.g., hands, feet) compared to torso load placement (Browning et al., 2007; Schertzer and Riemer, 2014). Possibly, the expectation that kinetic parameters would be sensitive to small position changes in centrally located loads was incorrect, or trained soldiers can tightly regulate joint energetics with different armor designs under constant loads. No other study has compared joint work and power profiles between different body armor designs while holding carried load constant. Thus, further research is required to substantiate our results. Additionally, future studies could systematically manipulate load placement (e.g., backpack or torso-borne) and examine the effects on mechanical work and power profiles.

Total and joint-level power magnitudes were largely determined by the amount of carried load and the walking speed. Our results suggest the effects of carried load and walking speed are largely additive, with greatest positive joint powers occurring with the fastest walking speed and heaviest carried load condition. These observations corroborate previous research demonstrating proportional increases in total and joint work with increased carried load (Grenier et al., 2012; Griffin et al., 2003; Huang and Kuo, 2014; Pierrynowsi et al., 1981). Additionally, incremental increases in carried load and walking speed have been shown to increase metabolic costs (Bastien et al., 2005), and confirm that the additional energy required for load carriage during walking is augmented at faster speeds.

As contracting muscle uses energy to generate power, analysis of joint energetics reveal which muscles/joints are responsible for greater metabolic cost. Our results showed $>80 \%$ of positive power during stance came from the hip and ankle during load carriage, which is consistent with previous studies using backpack loads (Brown et al., 2014) and in unloaded walking (Farris and Sawicki, 2012; Rubenson et al., 2011). Unique to this study, increasing carried load not only scaled joint power requirements but also shifted relative joint contributions to total positive power from ankle and knee towards the hip. We observed a $7.7 \%$ shift in contribution to the hip when carrying load and walking fast compared to walking with no load (Table 2). Additionally, regardless of load and walking speed condition, increases in total positive work were due primarily to increases in positive hip work, whereas proportional increases in positive knee and ankle work were not apparent (Figure 3). A proximal shift in power production may be due to the combined effects of adding load and placing the load posterior to the wearer's COM. Such a trade-off between a hip- or ankle-dominated power profile has been observed when participants were verbally instructed to modify ankle push-off force (Lewis and Ferris, 2008), and when assistive torques were externally applied to the hip (Lenzi et al., 2013), suggesting hip muscles (or an external actuator) compensated for reduced ankle plantarflexion power. We believe military boots are partly responsible for the observed shift, as they span the ankle and may reduce ankle range of motion, thereby reducing the ankle joint's ability to generate power. However, this conclusion has to be confirmed experimentally. Rather than saving energy, a proximal power shift may be a control strategy by which soldiers maintain forward progression without compromising stability during load carriage gait (Winter, 1995). 
Our results contrast with a previous study that reported the ankle contributed most to increased positive work demands of increased carried load (Huang and Kuo, 2014). However, directly comparing the above-cited study to ours is difficult as they had only eight civilian participants carrying backpack loads $<20 \mathrm{~kg}$ while walking at $1.25 \mathrm{~m} \cdot \mathrm{s}^{-1}$. Brown and colleagues (2014) tested heavier loads (i.e., 20-40kg) with soldiers and showed no change in joint contributions to positive power compared to carrying no load, although they examined walk-to-run transition instead of steady-state walking (Brown et al., 2014). Comparatively greater task demands in this study (carrying $30 \mathrm{~kg}$ and walking at $1.81 \mathrm{~m} \cdot \mathrm{s}^{-1}$ ) and using soldiers trained in load carriage may have caused a shift in power generation towards the hip. Further research over a broader range of walking speeds and loads with soldiers is recommended to elucidate changes in positive power contributions.

Total mechanical positive power was sensitive to changes in armor types and carried loads. Partly confirming our third hypothesis, hip joint contribution to total mechanical positive power was $5.2 \%$ more when carrying $30 \mathrm{~kg}$ of load compared to $15 \mathrm{~kg}$ while wearing CARM1, compensating for $2.4 \%$ and $2.8 \%$ reductions in knee and ankle contributions, respectively. However, no differences were observed between armor types while carrying the same loads. Conceivably, shoulder-to-hip load transfer did not change moment of inertia compared to TBAS, which would explain our lack of differences between armor types. Nevertheless, modulation of power production when wearing cARM1 with a $30 \mathrm{~kg}$ load suggests neuromuscular coordination strategies in carried-load contexts are design- and load-specific. Consequently, tailoring assistive devices to match the neuromuscular requirements of a particular load configuration identified in this study could help prolong soldier performance.

There were several limitations to this study that should be considered. First, knee abduction/adduction and internal/external rotations were solely determined from knee flexion/extension DOF using the same regression equation for all participants. This was done because measuring secondary knee motions from skin-surface marker data is error prone (Benoit et al., 2006). Second, joint work derived from inverse dynamics underestimates total musculotendon work (Neptune et al., 2009). Similar to Huang and Kuo (2014), total negative joint work per stride in our study ranged from 54.7-60.6\% of total positive joint work per stride for loads and walking speeds tested. This suggests a considerable amount of negative work performed by musculotendinous tissue deformation was not captured (Zelik and Kuo, 2012). Determining passive contributions to positive energy generation, and incorporating measures of muscle activity into a musculoskeletal model to estimate musculotendinous forces, power, and work would provide more representative estimations of actual work than those presented here, and may further highlight the importance of hip musculature to positive power generation in load carriage walking (Zelik et al., 2015).

In conclusion, this study was the first to demonstrate that different shoulder-to-hip load transfer devices do not alter magnitudes of mechanical joint work and power compared to a design with no load transfer mechanism. However, results showed joint contributions to positive power are design- and 
load-specific, meaning different load configurations may elicit different neuromuscular responses. To verify and extend our results, future studies could measure how joint powers and joint coordination change across a broader range of carried loads and walking speeds with more deliberate load perturbations. Additionally, understanding musculotendinous mechanics could lend deeper insight into strategies by which humans efficiently carry load.

\section{Acknowledgements}

The authors acknowledge Dr Jace Drain for his assistance with data collection along with the soldier participants who volunteered their time to participate.

\section{Conflict of interest statement and funding source}

The authors declare no conflicts of interest that could bias this research, including financial and/or personal relationships with other people or organizations. Defence Science and Technology Group contributed funding to support this research. They had no involvement into the study design, in the collection, analysis and interpretation of data; in the writing of the manuscript; and in the decision to submit the manuscript for publication. 
Figure 1. Relative contributions of hip, knee, and ankle to stance phase total mechanical positive power for the different load sharing systems and carried loads. *Indicates significant difference in the contribution of hip to total positive power compared to the value obtained with $15 \mathrm{~kg}$ of carried load for load distribution system.

Figure 2. Mean (lines) and standard deviation (shaded regions) joint angular velocities, moments, and powers for hip, knee, and ankle across the gait cycle (right foot strike to right foot strike) with different carried loads. Moments and power were normalized to body weight. Means were generated for all participants $(\mathrm{N}=2 \mathrm{O})$ and walking speeds. Arrows indicate locations in the gait cycle where significant differences in peak values between carried loads were found ( $\mathrm{p}<0.05)$. Vertical lines denote times of toeoff for different carried load conditions (follow same line style as curves).

Figure 3. Hip, knee, and ankle total positive work per stride versus total positive joint work per stride. Data points for moderate (i.e., green) and fast (i.e., red) walking speeds are distinguished by colors, whereas data points for $15 \mathrm{~kg}$ (i.e., circle) and $30 \mathrm{~kg}$ (i.e., triangle) of carried load are distinguished by shapes. A significant positive relationship was shown for hip and knee work ( $\mathrm{p}<0.05)$, whereas ankle work showed no relationship ( $\mathrm{p}>0.05$ ) to total positive work. A high correlation coefficient indicates that as total positive work increases, contribution from that joint also increases. The correlation coefficient displayed is the average of all data, as there were no significant differences between coefficients for individual carried load/walking speed combinations. 


\section{References}

Bastien, G.J., Willems, P.A., Schepens, B., Heglund, N.C., 2005. Effect of load and speed on the energetic cost of human walking. Eur. J. Appl. Physiol. 94, 76-83.

Beekley, M.D., Alt, J., Buckley, C.M., Duffey, M., Crowder, T.A., 2007. Effects of heavy load carriage during constant-speed, simulated, road marching. Mil. Med. 172, 592-595.

Benjamini, Y., Hochberg, Y., 1995. Controlling the False Discovery Rate - a Practical and Powerful Approach to Multiple Testing. J. R. Stat. Soc. Series. B Stat. Methodol. 57, 289-300.

Benoit, D.L., Ramsey, D.K., Lamontagne, M., Xu, L., Wretenberg, P., Renstrom, P., 2006. Effect of skin movement artifact on knee kinematics during gait and cutting motions measured in vivo. Gait Posture 24, 152-164.

Blacker, S.D., Fallowfield, J.L., Bilzon, J.L.J., Willems, M.E.T., 2013. Neuromuscular Impairment Following Backpack Load Carriage. J. Hum. Kinet. 37, 91.

Brown, T.N., O’Donovan, M., Hasselquist, L., Corner, B.D., Schiffman, J.M., 2014. Body borne loads impact walk-to-run and running biomechanics. Gait Posture 40, 237-242.

Browning, R.C., Modica, J.R., Kram, R., Goswami, A., 2007. The effects of adding mass to the legs on the energetics and biomechanics of walking. Med. Sci. Sports Exerc. 39, 515.

Buuren, S., Groothuis-Oudshoorn, K., 2011. mice: Multivariate imputation by chained equations in R. J. Stat. Soft. 45 .

Delp, S., Anderson, F.C., Arnold, A.S., Loan, P., Habib, A., John, C.T., Guendelman, E., Thelen, D.G., 2007. OpenSim: open-source software to create and analyze dynamic simulations of movement. IEEE Trans. Biomed. Eng. 54, 1940-1950.

Devroey, C., Jonkers, I., de Becker, A., Lenaerts, G., Spaepen, A., 2007. Evaluation of the effect of backpack load and position during standing and walking using biomechanical, physiological and subjective measures. Ergonomics 50, 728-742.

Donelan, J.M., Kram, R., Kuo, A.D., 2002. Mechanical work for step-to-step transitions is a major determinant of the metabolic cost of human walking. J. Exp. Biol. 205, 3717-3727.

Dorn, T.W., Wang, J.M., Hicks, J.L., Delp, S.L., 2015. Predictive simulation generates human adaptations during loaded and inclined walking. PLoS ONE 10, eo121407.

Farris, D.J., Sawicki, G.S., 2012. The mechanics and energetics of human walking and running: a joint level perspective. J. R. Soc. Interface 9, 110-118.

Grenier, J.G., Peyrot, N., Castells, J., Oullion, R., Messonnier, L., Morin, J.-B., 2012. Energy cost and mechanical work of walking during load carriage in soldiers. Med. Sci. Sports Exerc. 44, 1131-1140.

Griffin, T.M., Roberts, T.J., Kram, R., 2003. Metabolic cost of generating muscular force in human walking: insights from load-carrying and speed experiments. J. Appl. Physiol. 95, 172-183.

Hoaglin, D.C., Iglewicz, B., Tukey, J.W., 1986. Performance of some resistant rules for outlier labeling. J. Am. Stat. Assoc. 81, 991-999. 
Huang, T.-W.P., Kuo, A.D., 2014. Mechanics and energetics of load carriage during human walking. J. Exp. Biol. 217, 605-613.

Johnson, R.F., Knapik, J.J., Merullo, D.J., 1995. Symptoms during load carrying: effects of mass and load distribution during a 20-km road march. Percept. Mot. Skills 81, 331-338.

Knapik, J., Ang, P., Meiselman, H., Johnson, W., Kirk, J., Bensel, C., Hanlon, W., 1997. Soldier performance and strenuous road marching: influence of load mass and load distribution. Mil. Med, 162, $62-67$.

Lenton, G.K., Doyle, T.L.A., Saxby, D.J., Billing, D., Higgs, J., Lloyd, D.G., 2018. Integrating a hip belt with body armor reduces the magnitude and changes the location of shoulder pressure and perceived discomfort in soldiers. Ergonomics 61, 566-575.

Lenton, G.K., Doyle, T.L.A., Saxby, D.J., Lloyd, D.G., 2017. An alternative whole-body marker set to accurately and reliably quantify joint kinematics during load carriage. Gait Posture 54, 318-324.

Lenzi, T., Carrozza, M.C., Agrawal, S.K., 2013. Powered hip exoskeletons can reduce the user's hip and ankle muscle activations during walking. IEEE Trans. Neural Syst. Rehabil. Eng. 21, 938-948.

Lewis, C.L., Ferris, D.P., 2008. Walking with increased ankle pushoff decreases hip muscle moments. J. Biomech. 41, 2082-2089.

Lichtwark, G.A., Wilson, A.M., 2008. Optimal muscle fascicle length and tendon stiffness for maximising gastrocnemius efficiency during human walking and running. J. Theor. Biol. 252, 662-673.

Liew, B.X., Morris, S., Netto, K., 2016. The effects of load carriage on joint work at different running velocities. J. Biomech. 49, 3275-3280.

Mantoan, A., Pizzolato, C., Sartori, M., Sawacha, Z., Cobelli, C., Reggiani, M., 2015. MOtoNMS: A MATLAB toolbox to process motion data for neuromusculoskeletal modeling and simulation. Source Code Biol. Med. 10, 12.

Neptune, R.R., McGowan, C., Kautz, S., 2009. Forward dynamics simulations provide insight into muscle mechanical work during human locomotion. Exerc. Sport Sci. Rev. 37, 203.

Pierrynowsi, M.R., Norman, R.W., Winter, D.A., 1981. Mechanical energy analyses of the human during load carriage on a treadmill. Ergonomics 24, 1-14.

Quesada, P.M., Mengelkoch, L.J., Hale, R.C., Simon, S.R., 200o. Biomechanical and metabolic effects of varying backpack loading on simulated marching. Ergonomics 43, 293-309.

Rajagopal, A., Dembia, C.L., DeMers, M.S., Delp, D.D., Hicks, J.L., Delp, S.L., 2016. Full-Body Musculoskeletal Model for Muscle-Driven Simulation of Human Gait. IEEE Trans. Biomed. Eng. 63, 2068-2079.

Reinbolt, J.A., Schutte, J.F., Fregly, B.J., Koh, B.I., Haftka, R.T., George, A.D., Mitchell, K.H., 2005. Determination of patient-specific multi-joint kinematic models through two-level optimization. J. Biomech. 38, 621-626.

Richardson, J.T., 2011. Eta squared and partial eta squared as measures of effect size in educational research. Educ. Res. Rev. 6, 135-147. 
Robertson, G., Caldwell, G., Hamill, J., Kamen, G., Whittlesey, S., 2013. Research Methods in Biomechanics, 2E. Human Kinetics.

Robertson, G., Dowling, J.J., 2003. Design and responses of Butterworth and critically damped digital filters. J. Electromyogr. Kinesiol. 13, 569-573.

Royston, P., 2004. Multiple imputation of missing values. Stata journal 4, 227-241.

Rubenson, J., Lloyd, D.G., Heliams, D.B., Besier, T.F., Fournier, P.A., 2011. Adaptations for economical bipedal running: the effect of limb structure on three-dimensional joint mechanics. J. Royal Soc. Interface 8, 740 .

Schache, A.G., Brown, N.A., Pandy, M.G., 2015. Modulation of work and power by the human lowerlimb joints with increasing steady-state locomotion speed. J. Exp. Biol. 218, 2472-2481.

Schertzer, E., Riemer, R., 2014. Metabolic rate of carrying added mass: a function of walking speed, carried mass and mass location. Appl. Ergon. 45, 1422-1432.

Umberger, B.R., Martin, P.E., 2007. Mechanical power and efficiency of level walking with different stride rates. J. Exp. Biol. 210, 3255-3265.

Umberger, B.R., Rubenson, J., 2011. Understanding muscle energetics in locomotion: new modeling and experimental approaches. Exerc. Sport Sci. Rev. 39, 59-67.

Walker, P., Rovick, J., Robertson, D., 1988. The effects of knee brace hinge design and placement on joint mechanics. J. Biomech. 21, 965969-967974.

Wang, H., Frame, J., Ozimek, E., Leib, D., Dugan, E.L., 2013. The effects of load carriage and muscle fatigue on lower-extremity joint mechanics. Res. Q. Exerc. Sport 84, 305-312.

Winter, D.A., 1987. The biomechanics and motor control of human gait. University of Waterloo Press.

Winter, D.A., 1995. Human balance and posture control during standing and walking. Gait Posture 3, 193-214.

Winter, D.A., Eng, P., 1995. Kinetics: our window into the goals and strategies of the central nervous system. Behav. Brain Res. 67, 111-120.

Winter, D.A., Ruder, G.K., MacKinnon, C.D., 1990. Control of balance of upper body during gait, Multiple muscle systems. Springer, pp. 534-541.

Zelik, K.E., Kuo, A.D., 2012. Mechanical work as an indirect measure of subjective costs influencing human movement. PLoS ONE 7, e31143-e31143.

Zelik, K.E., Takahashi, K.Z., Sawicki, G.S., 2015. Six degree-of-freedom analysis of hip, knee, ankle and foot provides updated understanding of biomechanical work during human walking. J. Exp. Biol. 218, 876-886. 


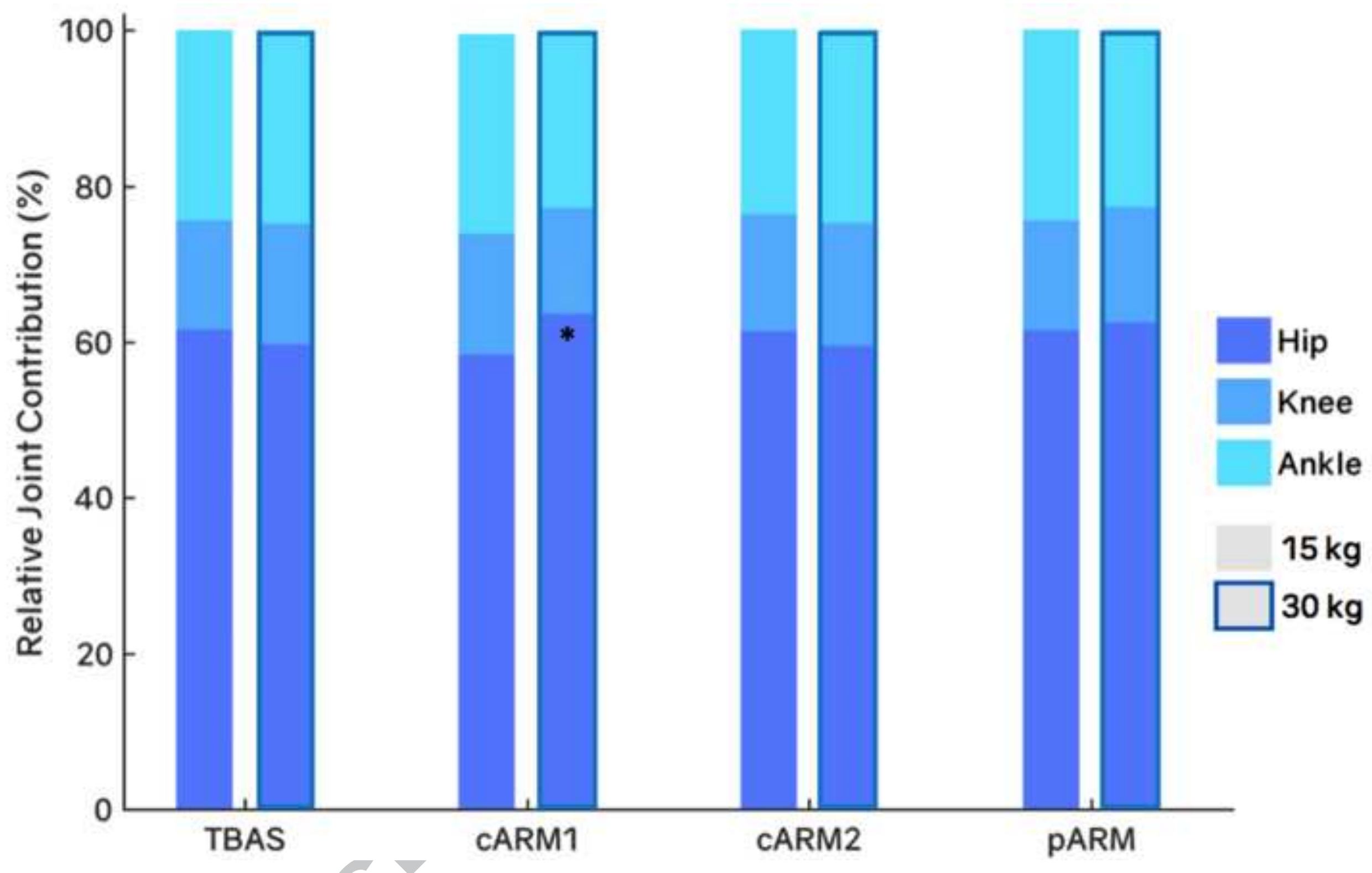




\section{ACCEPTED MANUSCRIPT}

Hip
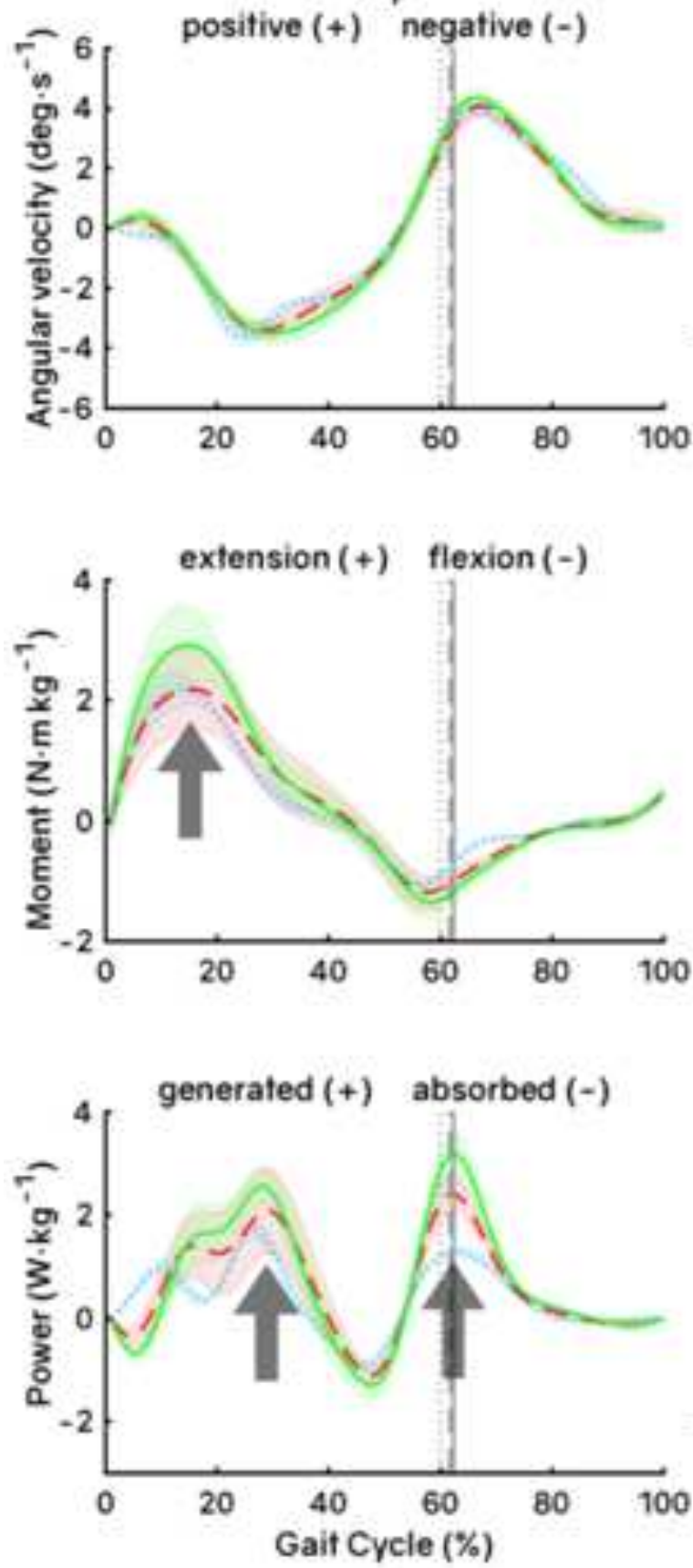

Knee
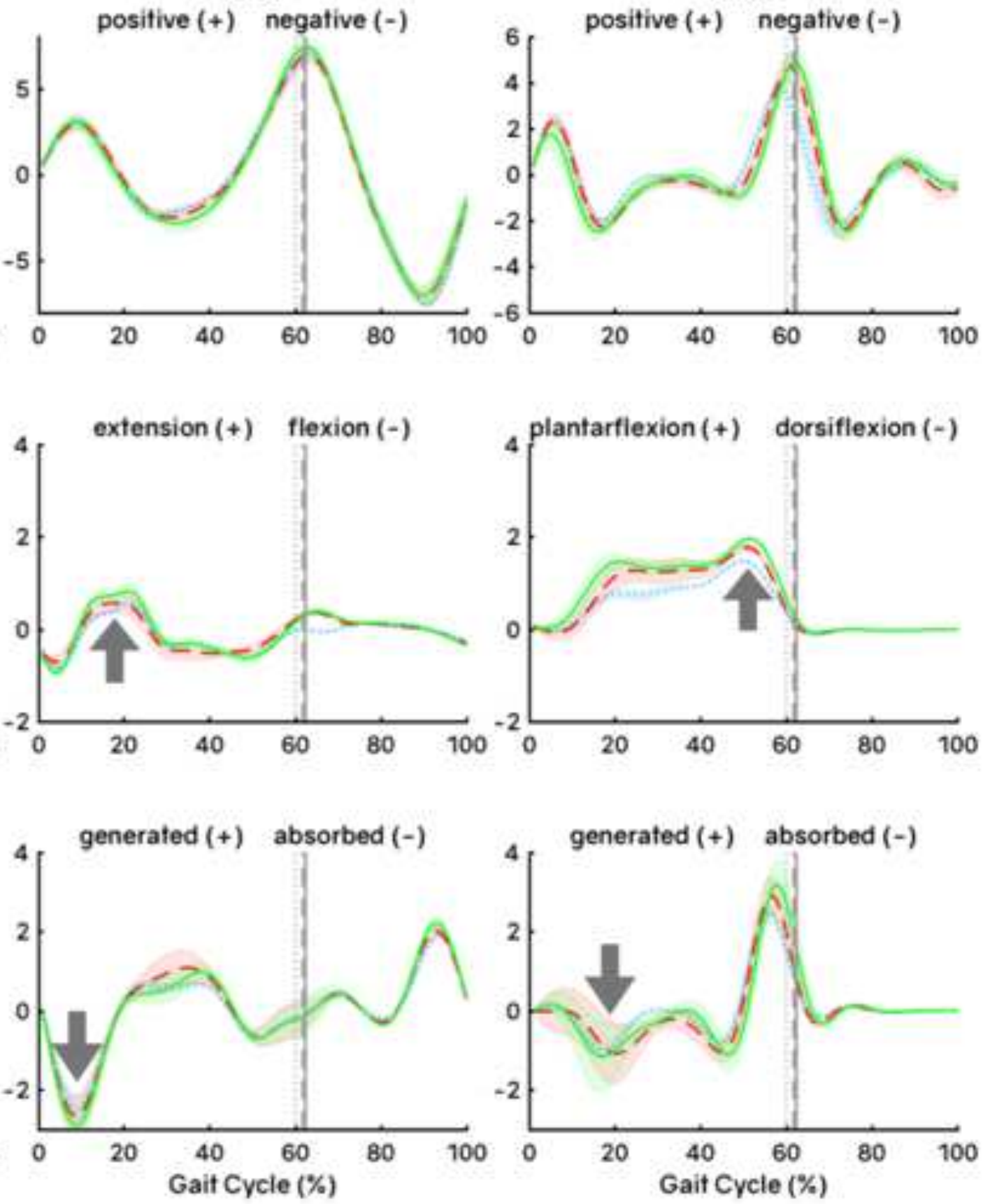

No load $--15 \mathrm{~kg}-30 \mathrm{~kg}$

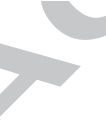



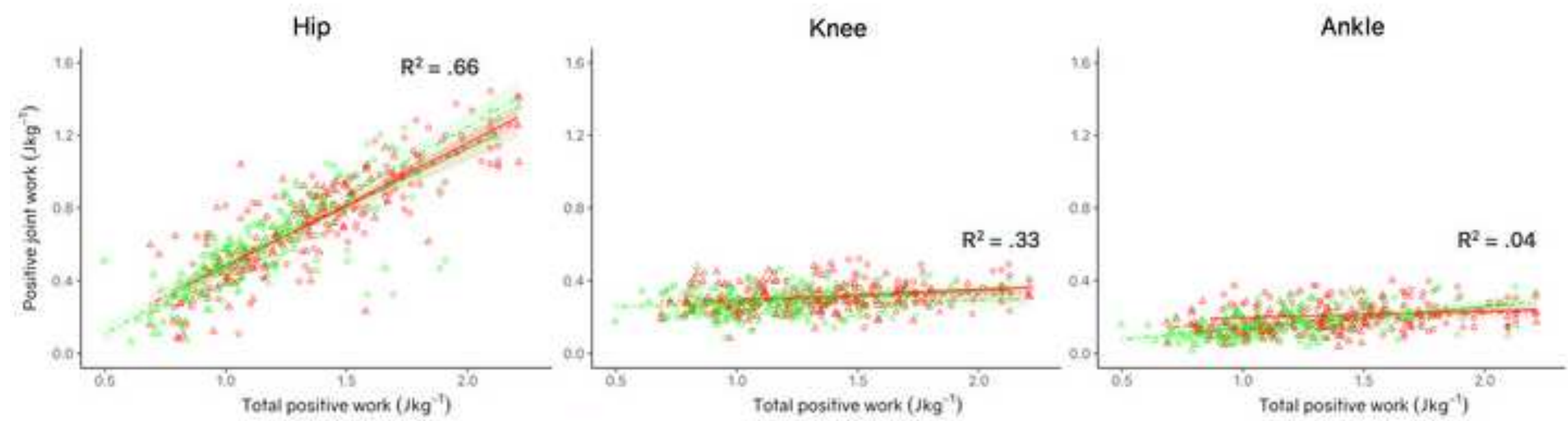

A $15 \mathrm{~kg}$ and moderate speed $-15 \mathrm{~kg}$ and fast speed
A $30 \mathrm{~kg}$ and moderate speed $-30 \mathrm{~kg}$ and fast speed 
Table 1. Mean \pm standard deviation magnitudes for joint work variables in stance and swing phases comparing main effects of load sharing systems. All values are reported as $\mathrm{Jkg}^{-1}$. Data for each load sharing system were pooled for walking speeds and load magnitudes.

\begin{tabular}{|c|c|c|c|c|c|}
\hline & \multicolumn{4}{|c|}{ Armor system } & \multirow{2}{*}{$\begin{array}{l}\text { Effect size } \\
\left(\text { Partial } \eta^{2}\right)\end{array}$} \\
\hline & TBAS & cARMi & cARM2 & pARM & \\
\hline Positive work stance & $1.28 \pm 0.33$ & $1.35 \pm 0.38$ & $1.28 \pm 0.37$ & $1.27 \pm 0.33$ & 0.012 \\
\hline Negative work stance* & $0.70 \pm 0.20$ & $0.75 \pm 0.22$ & $0.76 \pm 0.21$ & $0.77 \pm 0.20$ & 0.023 \\
\hline Positive hip work stance* & $0.68 \pm 0.27$ & $0.75 \pm 0.32$ & $0.65 \pm 0.29$ & $0.67 \pm 0.28$ & 0.023 \\
\hline Positive knee work stance & $0.18 \pm 0.08$ & $0.17 \pm 0.07$ & $0.18 \pm 0.08$ & $0.18 \pm 0.08$ & 0.004 \\
\hline Positive ankle work stance & $0.31 \pm 0.08$ & $0.31 \pm 0.06$ & $0.30 \pm 0.09$ & $0.29 \pm 0.07$ & 0.009 \\
\hline Positive work swing* & $0.22 \pm 0.05$ & $0.24 \pm 0.06^{1}$ & $0.24 \pm 0.06^{1}$ & $0.25 \pm 0.05^{1}$ & 0.055 \\
\hline Positive hip work swing* & $0.18 \pm 0.05$ & $0.21 \pm 0.06$ & $0.21 \pm 0.05$ & $0.22 \pm 0.04^{1}$ & 0.060 \\
\hline
\end{tabular}

${ }^{*}$ Indicates a significant main effect of armor $(\mathrm{p}<0.05)$. ${ }^{1}$ Indicates variable significantly increased in magnitude compared to values obtained for TBAS ( $\mathrm{p}<0.05)$.

Table 2. Mean \pm standard deviation magnitudes for joint work variables comparing main effects of walking speed and carried load. Data were pooled across the difference armor systems.

\begin{tabular}{|c|c|c|c|c|c|c|c|}
\hline \multirow{2}{*}{ Variable } & \multicolumn{2}{|c|}{ Walking speed } & \multirow{2}{*}{$\begin{array}{c}\text { Effect } \\
\text { Size } \\
\text { Partial } \eta^{2}\end{array}$} & \multicolumn{3}{|c|}{ Carried load } & \multirow{2}{*}{$\begin{array}{c}\text { Effect } \\
\text { Size } \\
\text { Partial } \eta^{2}\end{array}$} \\
\hline & & Fast & & No load & $15 \mathrm{~kg}$ & $30 \mathrm{~kg}$ & \\
\hline Positive work stance* & $1.15 \pm 0.32$ & $1.43 \pm 0.32^{1}$ & 0.174 & $0.91 \pm 0.27$ & $1.19 \pm 0.31^{2}$ & $1.38 \pm 0.35^{23}$ & 0.193 \\
\hline Negative work sta & $0.72 \pm 0.20$ & $0.78 \pm 0.22^{1}$ & 0.022 & $0.52 \pm 0.16$ & $0.68 \pm 0.18^{2}$ & $0.82 \pm 0.21^{23}$ & 0.122 \\
\hline Positive hip work stance* & $0.66 \pm 0.28$ & $0.84 \pm 0.27^{1}$ & 0.131 & $0.51 \pm 0.19$ & $0.69 \pm 0.27^{2}$ & $0.81 \pm 0.30^{23}$ & 0.071 \\
\hline $\begin{array}{l}\text { Positive knee work } \\
\text { stance* }^{*}\end{array}$ & $0.16 \pm 0.07$ & $0.20 \pm 0.08^{1}$ & 0.059 & $0.16 \pm 0.09$ & $0.17 \pm 0.07^{2}$ & $0.20 \pm 0.08^{23}$ & 0.052 \\
\hline $\begin{array}{l}\text { Positive ankle work } \\
\text { stance* }^{*}\end{array}$ & $0.29 \pm 0.07$ & $0.31 \pm 0.08^{1}$ & 0.035 & $0.24 \pm 0.07$ & $0.29 \pm 0.07^{2}$ & $0.31 \pm 0.08^{23}$ & 0.037 \\
\hline Positive work swing* & $0.22 \pm 0.05$ & $0.26 \pm 0.05^{1}$ & 0.101 & $0.18 \pm 0.05$ & $0.22 \pm 0.05^{2}$ & $0.26 \pm 0.05^{23}$ & 0.175 \\
\hline Positive hip work swing* & $0.20 \pm 0.05$ & $0.22 \pm 0.05^{1}$ & 0.032 & $0.15 \pm 0.04$ & $0.19 \pm 0.05^{2}$ & $0.23 \pm 0.05^{23}$ & 0.148 \\
\hline
\end{tabular}


${ }^{*}$ Indicates a significant main effect of speed and mass ( $\left.\mathrm{p}<0.05\right)$. ${ }^{1}$ Indicates significant difference from moderate speed $(\mathrm{p}<0.05) .{ }^{2}$ Indicates significant difference from no load. ${ }^{3}$ indicates significant difference from $15 \mathrm{~kg}$. 


\section{Accepted Manuscript}

Lower-limb joint work and power are modulated during load carriage based on load configuration and walking speed

Gavin K. Lenton, Tim L.A. Doyle, David G. Lloyd, Jeremy Higgs, Daniel Billing, David J. Saxby

PII: S0021-9290(18)30871-6

DOI: https://doi.org/10.1016/j.jbiomech.2018.11.036

Reference: BM 8945

To appear in:

Journal of Biomechanics

Received Date:

11 November 2017

Revised Date:

26 September 2018

Accepted Date:

23 November 2018

Please cite this article as: G.K. Lenton, T.L.A. Doyle, D.G. Lloyd, J. Higgs, D. Billing, D.J. Saxby, Lower-limb joint work and power are modulated during load carriage based on load configuration and walking speed, Journal of Biomechanics (2018), doi: https://doi.org/10.1016/j.jbiomech.2018.11.036

This is a PDF file of an unedited manuscript that has been accepted for publication. As a service to our customers we are providing this early version of the manuscript. The manuscript will undergo copyediting, typesetting, and review of the resulting proof before it is published in its final form. Please note that during the production process errors may be discovered which could affect the content, and all legal disclaimers that apply to the journal pertain. 